\title{
New Results on Generalized Graph Coloring
}

\author{
Vladimir E. Alekseev ${ }^{1}$ and Alastair Farrugia ${ }^{2}$ and Vadim V. Lozin ${ }^{3}$ \\ ${ }^{1}$ Department of Mathematical Logic, University of Nizhny Novgorod, Gagarina 23, 603950 RUSSIA. \\ E-mail: ave@uic.nnov.ru \\ ${ }^{2}$ Department of Combinatorics and Optimization, University of Waterloo, Waterloo, Ontario, Canada, N2L6G1. \\ E-mail: afarrugia@alumni.math.uwaterloo.ca \\ ${ }^{3}$ RUTCOR, Rutgers University, 640 Bartholomew Road, Piscataway, NJ 08854-8003, USA. \\ E-mail: lozinerutcor.rutgers.edu
}

received 25 Apr 2003, revised 02 Feb 2004, accepted 15 Feb 2004.

For graph classes $\mathcal{P}_{1}, \ldots, \mathcal{P}_{k}$, Generalized Graph Coloring is the problem of deciding whether the vertex set of a given graph $G$ can be partitioned into subsets $V_{1}, \ldots, V_{k}$ so that $V_{j}$ induces a graph in the class $\mathcal{P}_{j}(j=1,2, \ldots, k)$. If $\mathcal{P}_{1}=\cdots=\mathcal{P}_{k}$ is the class of edgeless graphs, then this problem coincides with the standard vertex $k$-COLORABILITY, which is known to be NP-complete for any $k \geq 3$. Recently, this result has been generalized by showing that if all $\mathcal{P}_{i}$ 's are additive hereditary, then the generalized graph coloring is NP-hard, with the only exception of bipartite graphs. Clearly, a similar result follows when all the $\mathcal{P}_{i}$ 's are co-additive.

In this paper, we study the problem where we have a mixture of additive and co-additive classes, presenting several new results dealing both with NP-hard and polynomial-time solvable instances of the problem.

Keywords: Generalized Graph Coloring; Polynomial algorithm; NP-completeness

\section{Introduction}

All graphs in this paper are finite, without loops and multiple edges. For a graph $G$ we denote by $V(G)$ and $E(G)$ the vertex set and the edge set of $G$, respectively. By $N(v)$ we denote the neighborhood of a vertex $v \in V(G)$, i.e. the subset of vertices of $G$ adjacent to $v$. The subgraph of $G$ induced by a set $U \subseteq V(G)$ will be denoted $G[U]$. We say that a graph $G$ is $H$-free if $G$ does not contain $H$ as an induced subgraph. As usual, $K_{n}$ and $P_{n}$ stand for the complete graph and chordless path on $n$ vertices, respectively, and the complement of a graph $G$ is denoted $\bar{G}$.

An isomorphism-closed class of graphs, or synonymously graph property, $\mathcal{P}$ is said to be hereditary [2] if $G \in \mathcal{P}$ implies $G-v \in \mathcal{P}$ for any vertex $v \in V(G)$. We call $\mathcal{P}$ monotone if $G \in \mathcal{P}$ implies $G-v \in \mathcal{P}$ for any vertex $v \in V(G)$ and $G-e \in \mathcal{P}$ for any edge $e \in E(G)$. This terminology has been used by other authors too, but it is not standard; in particular, some papers use "hereditary" for the properties that we call "monotone". Clearly every monotone property is hereditary, but the converse statement is not true in general. A property $\mathcal{P}$ is additive if $G_{1} \in \mathcal{P}$ and $G_{2} \in \mathcal{P}$ with $V\left(G_{1}\right) \cap V\left(G_{2}\right)=\emptyset$ implies $G=$ $\left(V\left(G_{1}\right) \cup V\left(G_{2}\right), E\left(G_{1}\right) \cup E\left(G_{2}\right)\right) \in \mathcal{P}$. The class of graphs containing no induced subgraphs isomorphic 
to graphs in a set $Y$ will be denoted $\operatorname{Free}(Y)$. It is well known that a class of graphs $\mathcal{P}$ is hereditary if and only if $\mathcal{P}=\operatorname{Free}(Y)$ for some set $Y$.

A property is said to be non-trivial if it contains at least one, but not all graphs. The complementary property of $\mathcal{P}$ is $\overline{\mathcal{P}}:=\{\bar{G} \mid G \in \mathcal{P}\}$. Note that $\mathcal{P}$ is hereditary if and only if $\overline{\mathcal{P}}$ is. So a co-additive hereditary property, i.e. the complement of an additive hereditary property, is itself hereditary.

Let $\mathcal{P}_{1}, \ldots, \mathcal{P}_{k}$ be graph properties with $k>1$. A graph $G=(V, E)$ is $\left(\mathcal{P}_{1}, \ldots, \mathcal{P}_{k}\right)$-colorable if there is a partition $\left(V_{1}, \ldots, V_{k}\right)$ of $V(G)$ such that $G\left[V_{j}\right] \in \mathcal{P}_{j}$ for each $j=1, \ldots, k$. The problem of recognizing $\left(\mathcal{P}_{1}\right.$, $\ldots, \mathcal{P}_{k}$ )-colorable graphs is usually referred to as Generalized Graph Coloring [8]. When $\mathcal{P}_{1}=\cdots=\mathcal{P}_{k}$ is the class $O$ of edgeless graphs, this problem coincides with the standard $k$-COLORABILITY, which is known to be NP-complete for $k \geq 3$. Generalized Graph Coloring remains difficult for many other cases. For example, Cai and Corneil [10] showed that $\left(\right.$ Free $\left(K_{n}\right)$,Free $\left(K_{m}\right)$ )-coloring is NP-complete for any integers $m, n \geq 2$, with the exception $m=n=2$. Important NP-completeness results were obtained by Brown [8] and Achlioptas [1] (when the $\mathcal{P}_{i}$ 's are identical), and Kratochvíl and Schiermeyer [18] (when the $\mathcal{P}_{i}$ 's may be different) (see [2] for more results on this topic). These lead to the following recent generalization [11]:

Theorem 1 If $\mathcal{P}_{1}, \ldots, \mathcal{P}_{k}(k>1)$ are additive hereditary properties of graphs, then the problem of recognizing $\left(\mathcal{P}_{1}, \ldots, \mathcal{P}_{k}\right)$-colorable graphs is $N P$-hard, unless $k=2$ and $\mathcal{P}_{1}=\mathscr{P}_{2}$ is the class of edgeless graphs.

Clearly, a similar result follows for co-additive properties. In the present paper we focus on the case where we have a mixture of additive and co-additive properties.

The product of graph properties $\mathcal{P}_{1}, \ldots, \mathcal{P}_{k}$ is $\mathcal{P}_{1} \circ \ldots \circ \mathcal{P}_{k}:=\left\{G \mid G\right.$ is $\left(\mathscr{P}_{1}, \ldots, \mathscr{P}_{k}\right)$-colorable $\}$. A property is reducible if it is the product of two other properties, otherwise it is irreducible [3]. It can be easily checked that the product of additive hereditary (or monotone) properties is again additive hereditary (respectively, monotone); and that $\overline{\mathcal{P}_{1} \circ \cdots \circ \mathcal{P}_{k}}=\overline{\mathcal{P}_{1}} \circ \cdots \circ \overline{\mathcal{P}_{k}}$. So, without loss of generality we shall restrict our study to the case $k=2$ and shall denote throughout the paper an additive property by $\mathcal{P}$ and co-additive by $Q$. We will refer to the problem of recognizing $(\mathcal{P}, Q)$-colorable graphs as $(\mathcal{P} \circ Q)$ RECOGNITION.

The plan of the paper is as follows. In Section 2 , we show that $(\mathcal{P} \circ Q)$-RECOGNITION cannot be simpler than $\mathcal{P}$ - or $Q$-RECOGNITION. In particular, we prove that $(\mathcal{P} \circ Q)$-RECOGNITION is NP-hard whenever $\mathcal{P}$ - or $Q$-RECOGNITION is NP-hard. Then, in Section 3 , we study the problem under the assumption that both $\mathcal{P}$ - and $Q$-RECOGNITION are polynomial-time solvable and present infinitely many classes of $(\mathcal{P}, Q)$-colorable graphs with polynomial recognition time. These two results together give a complete answer to the question of complexity of $(\mathcal{P} \circ Q)$-RECOGNITION when $\mathcal{P}$ and $\bar{Q}$ are additive monotone. When $\mathcal{P}$ and $\bar{Q}$ are additive hereditary (but not both monotone), there remains an unexplored gap that we discuss in the concluding section of the paper.

\section{NP-hardness}

In this section we prove that if $\mathcal{P}$-RECOGNITION (or $Q$-RECOGNITION) is NP-hard, then so is $(\mathcal{P} \circ Q)$ RECOGNITION. This is a direct consequence of the theorem below. In this theorem we use uniquely colorable graphs, which are often a crucial tool in proving coloring results.

A graph $G$ is uniquely $\left(\mathcal{P}_{1}, \ldots, \mathcal{P}_{k}\right)$-colorable if $\left(V_{1}, \ldots, V_{k}\right)$ is its only $\left(\mathcal{P}_{1}, \ldots, \mathcal{P}_{k}\right)$-partition, up to some permutation of the $V_{i}$ 's. If, say, $\mathcal{P}_{1}=\mathcal{P}_{2}$, then $\left(V_{2}, V_{1}, V_{3}, \ldots, V_{k}\right)$ will also be a $\left(\mathcal{P}_{1}, \mathcal{P}_{2}, \mathcal{P}_{3}, \ldots, \mathcal{P}_{k}\right)$-coloring 
of $G$; such a permutation (of $V_{i}$ 's that correspond to equal properties) is a trivial interchange. A graph is strongly uniquely $\left(\mathcal{P}_{1}, \ldots, \mathcal{P}_{k}\right)$-colorable if $\left(V_{1}, \ldots, V_{k}\right)$ is the only $\left(\mathcal{P}_{1}, \ldots, \mathcal{P}_{k}\right)$-coloring, up to trivial interchanges.

When $\mathcal{P}_{1}, \ldots, \mathcal{P}_{k}$ are irreducible hereditary properties, and each $\mathcal{P}_{i}$ is either additive or co-additive, there is a strongly uniquely $\left(\mathcal{P}_{1}, \ldots, \mathcal{P}_{k}\right)$-colorable graph with each $V_{i}$ non-empty. This important construction, for additive $\mathcal{P}_{i}$ 's, is due to Mihók [20], with some embellishments by Broere and Bucko [6]. The proof that these graphs are actually uniquely colorable follows from [7], [14, Thm. 5.3] or [13]. Obviously, similar results apply to co-additive properties. The generalization to mixtures of additive and co-additive properties can be found in [12, Cor. 4.3.6, Thm. 5.3.2]. For irreducible additive monotone properties, there is a much simpler proof of the existence of uniquely colorable graphs [21].

Theorem 2 Let $\mathcal{P}$ and $\bar{Q}$ be additive hereditary properties. Then there is a polynomial-time reduction from $\mathcal{P}$-RECOGNITION to $(\mathcal{P} \circ Q)$-RECOGNITION.

Proof. Let $\mathcal{P}=\mathcal{P}_{1} \circ \cdots \circ \mathcal{P}_{n}$ and $Q=Q_{\mathfrak{l}} \circ \cdots \circ Q_{r}$, where the $\mathcal{P}_{i}$ 's and $\bar{Q}_{j}$ 's are the irreducible additive hereditary factors whose existence is guaranteed by the unique factorization theorem [20, 13]. As noted above, there is a strongly uniquely $\left(\mathcal{P}_{1}, \ldots, \mathcal{P}_{n}, Q_{1}, \ldots, Q_{x}\right)$-colorable graph $H$ with partition $\left(U_{1}, \ldots, U_{n}, W_{1}, \ldots, W_{r}\right)$, where each $U_{i}$ and $W_{j}$ is non-empty. Define $U:=U_{1} \cup \cdots \cup U_{n}$ and $W:=$ $W_{1} \cup \cdots \cup W_{r}$. Arbitrarily fix a vertex $u \in U_{1}$, and define $N_{W}(u):=N(u) \cap W$. For any graph $G$, let the graph $G_{H}$ consist of disjoint copies of $G$ and $H$, together with edges $\left\{v w \mid v \in V(G), w \in N_{W}(u)\right\}$. We claim that $G_{H} \in \mathcal{P} \circ Q$ if and only if $G \in \mathcal{P}$.

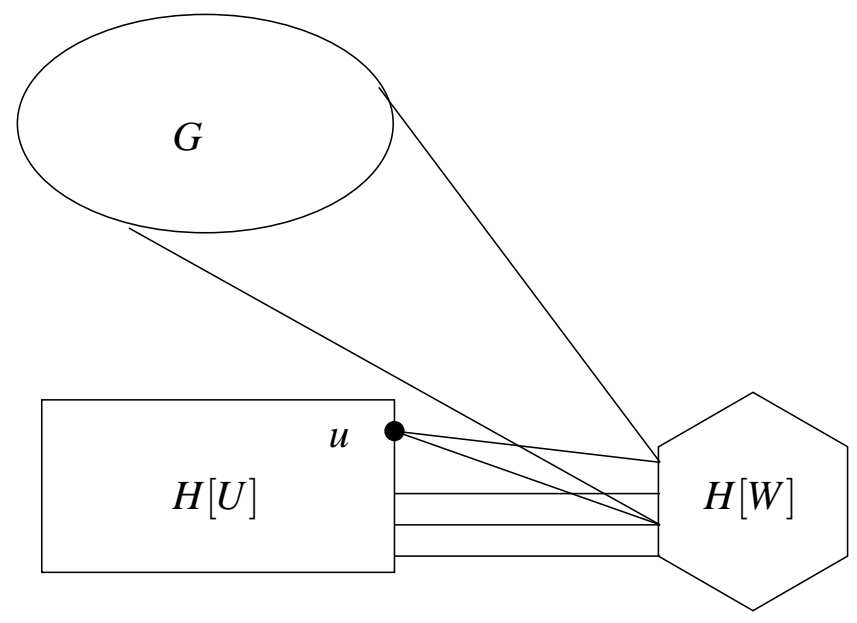

Fig. 1: Using $H$ to construct $G_{H}$.

If $G \in \mathcal{P}$, then, by additivity, $G \cup H[U]$ is in $\mathcal{P}$, and thus $G_{H}$ is in $\mathcal{P} \circ Q$. Conversely, suppose $G_{H} \in \mathcal{P} \circ Q$, i.e. it has a $\left(\mathcal{P}_{1}, \ldots, \mathcal{P}_{n}, Q_{l}, \ldots, Q_{t}\right)$-partition, say $\left(X_{1}, \ldots, X_{n}, Y_{1}, \ldots, Y_{r}\right)$. Since $H$ is strongly uniquely partitionable, we can assume that, for $1 \leq i \leq r, Y_{i} \cap V(H)=W_{i}$. Now, suppose for contradiction that, for some $k$, there is a vertex $v \in V(G)$ such that $v \in Y_{k}$; without loss of generality, let $k=r$. Then $G_{H}\left[W_{r} \cup\{v\}\right] \cong H\left[W_{r} \cup\{u\}\right]$ is in $Q_{r}$, so $\left(U_{1} \backslash\{u\}, U_{2}, \ldots, U_{n}, W_{1}, \ldots, W_{r-1}, W_{r} \cup\{u\}\right)$ is a new 
$\left(\mathcal{P}_{1}, \ldots, \mathcal{P}_{n}, Q_{l}, \ldots, Q_{v}\right)$-partition of $H$, which is impossible. Thus, $V(G) \subseteq X_{1} \cup \cdots \cup X_{n}$, and hence $G \in \mathcal{P}$, as claimed.

Since $H$ is a fixed graph, $G_{H}$ can be constructed in time linear in $|V(G)|$, so the theorem is proved.

\section{Polynomial time results}

Lemma 1 For any $\mathcal{P} \subseteq$ Free $\left(K_{n}\right)$ and $Q \subseteq$ Free $\left(\bar{K}_{m}\right)$, there exists a constant $\tau=\tau(\mathcal{P}, Q)$ such that for every graph $G=(V, E) \in \mathcal{P} \circ Q$ and every subset $B \subseteq V$ with $G[B] \in \mathcal{P}$, at least one of the following statements holds:

(a) there is a subset $A \subseteq V$ such that $G[A] \in \mathcal{P}, G[V-A] \in \mathcal{Q}$ and $|A-B| \leq \tau$,

(b) there is a subset $C \subseteq V$ such that $G[C] \in \mathcal{P},|C|=|B|+1$, and $|B-C| \leq \tau$.

Proof. By the Ramsey Theorem [17], for each positive integers $m$ and $n$, there is a constant $R(m, n)$ such that every graph with more than $R(m, n)$ vertices contains either a $\bar{K}_{m}$ or a $K_{n}$ as an induced subgraph. For two classes $\mathcal{P} \subseteq$ Free $\left(K_{n}\right)$ and $Q \subseteq$ Free $\left(\bar{K}_{m}\right)$, we define $\tau=\tau(\mathcal{P}, Q)$ to be equal $R(m, n)$. Let us show that with this definition the proposition follows.

Let $G=(V, E)$ be a graph in $\mathcal{P} \circ Q$, and $B$ a subset of $V$ such that $G[B] \in \mathcal{P}$. Consider an arbitrary subset $A \subseteq V$ such that $G[A] \in \mathcal{P}$ and $G[V-A] \in Q$. If (a) does not hold, then $|A-B|>\tau$. Furthermore, $G[B-A] \in \mathcal{P} \cap Q \subseteq$ Free $\left(K_{n}, \bar{K}_{m}\right)$, and hence $|B-A| \leq \tau$. Therefore, $|A|>|B|$. But then any subset $C \subseteq A$ such that $A \cap B \subseteq C$ and $|C|=|B|+1$ satisfies (b).

Lemma 1 suggests the following recognition algorithm for graphs in the class $\mathcal{P} \circ Q$.

\section{Algorithm $\mathcal{A}$}

Input: A graph $G=(V, E)$.

Output: YES if $G \in \mathcal{P} \circ Q$, or NO otherwise.

(1) Find in $G$ any inclusion-wise maximal subset $B \subseteq V$ inducing a $K_{n}$-free graph.

(2) If there is a subset $C \subseteq V$ satisfying condition (b) of Lemma 1 , then set $B:=C$ and repeat Step (2).

(3) If $G$ contains a subset $A \subseteq V$ such that

$$
\begin{aligned}
& |B-A| \leq \tau, \\
& |A-B| \leq \tau, \\
& G[A] \in \mathcal{P}, \\
& G[V-A] \in Q,
\end{aligned}
$$

output YES, otherwise output NO.

Theorem 3 If graphs on $p$ vertices in a class $\mathcal{P} \subseteq$ Free $\left(K_{n}\right)$ can be recognized in time $O\left(p^{k}\right)$ and graphs in a class $Q \subseteq$ Free $\left(\bar{K}_{m}\right)$ can be recognized in time $O\left(p^{l}\right)$, then Algorithm $\mathcal{A}$ recognizes graphs on $p$ vertices in the class $\mathcal{P} \circ Q$ in time $O\left(p^{2 \tau+\max \{(k+2), \max \{k, l\}\}}\right)$, where $\tau=\tau(\mathcal{P}, Q)$. 
Proof. Correctness of the algorithm follows from Lemma 1. Now let us estimate its time complexity. In Step (2), the algorithm examines at most $\left(\begin{array}{c}p \\ \tau\end{array}\right)\left(\begin{array}{c}p \\ \tau+1\end{array}\right)$ subsets $C$ and for each of them verifies whether $G[C] \in \mathcal{P}$ in time $O\left(p^{k}\right)$. Since Step (2) loops at most $p$ times, its time complexity is $O\left(p^{2 \tau+k+2}\right)$. In Step (3), the algorithm examines at most $\left(\begin{array}{l}p \\ \tau\end{array}\right)^{2}$ subsets $A$, and for each $A$, it verifies whether $G[A] \in \mathcal{P}$ in time $O\left(p^{k}\right)$ and whether $G[V-A] \in Q$ in time $O\left(p^{l}\right)$. Summarizing, we conclude that the total time complexity of the algorithm is $O\left(p^{2 \tau+\max \{(k+2), \max \{k, l\}\}}\right)$.

Notice that Theorem 3 generalizes several positive results on the topic under consideration. For instance, the split graphs [16], which are $\left(\right.$ Free $\left(K_{2}\right)$, Free $\left.\left(\overline{K_{2}}\right)\right)$-colorable by definition, can be recognized in polynomial time. More general classes have been studied under the name of polar graphs in [9, 19, 22]. By definition, a graph is $(m-1, n-1)$ polar if it is $(\mathcal{P}, Q)$-colorable with $\mathcal{P}=$ Free $\left(K_{n}, P_{3}\right)$ and $Q=$ Free $\left(\bar{K}_{m}, \bar{P}_{3}\right)$. It is shown in [19] that for any particular values of $m \geq 2$ and $n \geq 2,(m-1, n-1)$ polar graphs on $p$ vertices can be recognized in time $O\left(p^{2 m+2 n+3}\right)$.

Further examples generalizing the split graphs were examined in [4] and [15], where the authors showed that classes of graphs partitionable into at most two independent sets and two cliques can be recognized in polynomial time. These are special cases of $(\mathcal{P} \circ Q)$-RECOGNITION with $\mathcal{P} \subseteq$ Free $\left(K_{3}\right)$ and $Q \subseteq$ $\operatorname{Free}\left(\bar{K}_{3}\right)$.

\section{Concluding results and open problems}

Theorems 2 and 3 together provide complete answer to the question of complexity of $(\mathcal{P} \circ Q)$-RECOGNITION in case of monotone properties $\mathcal{P}$ and $\bar{Q}$. Indeed, if $\mathcal{P}$ is an additive monotone non-trivial property, then $\mathcal{P} \subseteq \operatorname{Free}\left(K_{n}\right)$ for a certain value of $n$, since otherwise it includes all graphs. Similarly, if $\bar{Q}$ is additive monotone, then $Q \subseteq$ Free $\left(\bar{K}_{m}\right)$ for some $m$. Hence, the following theorem holds.

Theorem 4 If $\mathcal{P}$ and $\bar{Q}$ are additive monotone properties, then $(\mathcal{P} \circ Q)$-RECOGNITION has polynomialtime complexity if and only if $\mathcal{P}$ - and $Q$-RECOGNITION are both polynomial-time solvable; moreover, $(\mathcal{P} \circ Q)$-RECOGNITION is in NP if and only if $\mathcal{P}$ - and $Q$-RECOGNITION are both in NP.

If $\mathcal{P}$ and $\bar{Q}$ are general additive hereditary properties (not necessarily monotone), then there is an unexplored gap containing properties $\mathcal{P} \circ Q$, where $\mathcal{P}$ and $Q$ can both be recognized in polynomial time, but $\mathcal{K} \subset \mathcal{P}$ or $O \subset Q$ (where $\mathcal{K}:=\bar{O}$ is the set of cliques). In the rest of this section we show that this gap contains both NP-hard and polynomial-time solvable instances, and propose several open problems to study.

For a polynomial time result we refer the reader to [22], where the authors claim that $(\mathcal{P} \circ Q)$-RECOGNITION is polynomial-time solvable if $\mathcal{P}$ is the class of edgeless graphs and $Q=$ Free $\left(\bar{P}_{3}\right)$. Notice that Free $\left(\bar{P}_{3}\right)$ contains all edgeless graphs and hence Theorem 3 does not apply to this case. Interestingly enough, when we extend $\mathcal{P}$ to the class of bipartite graphs, we obtain an NP-hard instance of the problem, as the following theorem shows.

Theorem 5 If $\mathcal{P}$ is the class of bipartite graphs and $Q=$ Free $\left(\bar{P}_{3}\right)$, then $(\mathcal{P} \circ Q)$-RECOGNITION is NPhard.

Proof. We reduce the standard 3-COLORABILITY to our problem. Consider an arbitrary graph $G$ and let $G^{\prime}$ be the graph obtained from $G$ by adding a triangle $T=(1,2,3)$ with no edges between $G$ and $T$. We claim that $G$ is 3-colorable if and only if $G^{\prime}$ is $(\mathcal{P}, Q)$-colorable. 
First, assume that $G$ is 3-colorable and let $V_{1}, V_{2}, V_{3}$ be a partition of $V(G)$ into three independent sets. We define $V_{j}^{\prime}=V_{j} \cup\{j\}$ for $j=1,2,3$. Then $G^{\prime}\left[V_{1}^{\prime} \cup V_{2}^{\prime}\right]$ is a bipartite graph and $G^{\prime}\left[V_{3}^{\prime}\right] \in \operatorname{Free}\left(\bar{P}_{3}\right)$, and the proposition follows.

Conversely, let $U \cup W$ be a partition of $V\left(G^{\prime}\right)$ with $G^{\prime}[U]$ being a bipartite graph and $G^{\prime}[W] \in$ Free $\left(\bar{P}_{3}\right)$. Clearly, $T \nsubseteq \amalg$. If $T-U$ contains a single vertex, then $G^{\prime}[W-T]$ is an edgeless graph, since otherwise a $\bar{P}_{3}$ arises. If $T-U$ contains more than one vertex, then $W-T=\emptyset$ for the same reason. Clearly, in both cases $G$ is a 3 -colorable graph.

This discussion presents the natural question of exploring the boundary that separates polynomial from non-polynomial time solvable instances in the above-mentioned gap. As one of the smallest classes in this gap with unknown recognition time complexity, let us point out $(\mathcal{P}, Q)$-COLORABLE graphs with $\mathcal{P}=O$ and $Q=\operatorname{Free}\left(2 K_{2}, P_{4}\right)$, where $2 K_{2}$ is the disjoint union of two copies of $K_{2}$.

Another direction for prospective research deals with $(\mathcal{P}, Q)$-colorable graphs where $\mathcal{P}$ or $Q$ is neither additive nor co-additive. This area seems to be almost unexplored and also contains both NP-hard and polynomial-time solvable problems. To provide some examples, let $Q$ be the class of complete bipartite graphs, which is obviously neither additive nor co-additive. The class of graphs partitionable into an independent set and a complete bipartite graph has been studied in [5] under the name of bisplit graphs and has been shown there to be polynomial-time recognizable. Again, extension of $\mathcal{P}$ to the class of all bipartite graphs transforms the problem into an NP-hard instance.

Theorem 6 If $\mathcal{P}$ is the class of bipartite graphs and $Q$ is the class of complete bipartite graphs, then $(\mathcal{P} \circ Q)$-RECOGNITION is NP-hard.

Proof. The reduction is again from 3-COlORABILITy. For a graph $G$, we define $G^{\prime}$ to be the graph obtained from $G$ by adding a new vertex adjacent to every vertex of $G$. It is a trivial exercise to verify that $G$ is 3-colorable if and only if $G^{\prime}$ is $(\mathcal{P}, Q)$-COLORABLE.

\section{Acknowledgements}

The second author wishes to thank R. Bruce Richter, his doctoral supervisor, for his valuable comments; as well as the Canadian government, which is fully funding his studies in Waterloo through a Canadian Commonwealth Scholarship.

\section{References}

[1] D. Achlioptas, The complexity of $G$-free colorability, Discrete Math. 165/166 (1997) 21-30.

[2] M. Borowiecki, I. Broere, M. Frick, P. Mihók and G. Semanišin, Survey of hereditary properties of graphs, Discuss. Math. Graph Theory 17 (1997) 5-50.

[3] M. Borowiecki and P. Mıнóк, Hereditary properties of graphs, in V.R. Kulli, ed., Advances in Graph Theory (Vishwa International Publication, Gulbarga, 1991) 42-69.

[4] A. BRAndstäDT, V.B. LE, and T. SZYMCZAK, The complexity of some problems related to graph 3-colorability, Discrete Appl. Math. 89 (1998) 59-73. 
[5] A. BRandstädT, P.L. Hammer, V.B. LE and V.V. LozIn, Bisplit Graphs, DIMACS Technical Report 2002-44 (2002) Rutgers University (available on-line at http://dimacs.rutgers.edu/TechnicalReports/2002.html)

[6] I. BROERE and J. BuCKO, Divisibility in additive hereditary properties and uniquely partitionable graphs, Tatra Mt. Math. Publ. 18 (1999), 79-87.

[7] I. BRoERE, B. BuCKO and P. MiHók, Criteria of the existence of uniquely partionable graphs with respect to additive induced-hereditary properties, Discuss. Math. Graph Theory 22 (2002) 31-37.

[8] J. BROWN, The complexity of generalized graph colorings, Discrete Appl. Math. 69 (1996) 257-270.

[9] ZH. A. ChernyaK and A. A. Chernyak, About recognizing $(\alpha, \beta)$ classes of polar graphs, Discrete Math. 62 (1986) 133-138.

[10] L. CAI and D.G. CoRnEIL, A generalization of perfect graphs - i-perfect graphs, J. Graph Theory 23 (1996) 87-103.

[11] A. FARRUGia, Vertex-partitioning into fixed additive induced-hereditary properties is NP-hard, submitted to Electron. J. Combin.

[12] A. FArrugia, Uniqueness and complexity in generalised colouring. Ph.D. thesis, University of Waterloo, Waterloo, Ontario, Canada. February 2003. (available on-line at http://www.math.uwaterloo.ca/ afarrugia/thesis.ps or http://etheses.uwaterloo.ca)

[13] A. FArrugia, P. Mihók, R.B. Richter and G. SEMAnišin, Factorisations and characterisations of induced-hereditary and compositive properties, submitted.

[14] A. FARRUgia and R.B. Richter, Unique factorization of additive induced-hereditary properties, to appear in Discussiones Mathematicae Graph Theory.

[15] T. Feder, P. Hell, S. Klein and R. Motwani, Complexity of graph partition problems, ACM Symposium on the Theory of Computing (Atlanta, Georgia, USA, 1999) 464-472.

[16] S. Foldes and P.L. Hammer, Split graphs, Congres. Numer. 19 (1977) 311-315.

[17] R.L. Graham, B.L. Rothschild, and J.H. Spencer, Ramsey Theory, Wiley, New York, 1980.

[18] J. KRATOChVÍl and I. SCHIERMEYER, On the computational complexity of (O,P)-partition problems, Discussiones Mathematicae Graph Theory 17 2(1997), 253-258.

[19] O. MEL'NIKOV and P.P. KOZHICH, Algorithms for recognizing the polarity of a graph with bounded parameters, Izvestia Akad. Nauk BSSR, ser. Fiz. Mat. Nauk 6 (1985) 50-54 (in Russian).

[20] P. Мıнóк, Unique Factorization Theorem, Discussiones Mathematicae Graph Theory 20 (2000), 143-153.

[21] P. Minók, G. SEMANIŠIN and R. VASKY, Additive and hereditary properties of graphs are uniquely factorizable into irreducible factors, J. Graph Theory 33 (2000) 44-53.

[22] R.I. Tyshkevich and A.A. Chernyak, Algorithms for the canonical decomposition of a graph and recognizing polarity, Izvestia Akad. Nauk BSSR, ser. Fiz.-Mat. Nauk 6 (1985) 16-23 (in Russian). 
